\title{
A RATIONAL APPROACH TO DETERMINE MINIMUM STRENGTH THRESHOLDS IN NOVEL STRUCTURAL MATERIALS
}

\author{
Willi W. Schur, $\mathrm{PhD} *, \dagger$ \\ Physical Science Laboratory \\ New Mexico State University \\ Field Office: NASA-GSFC- Wallops Flight Facility \\ Wallops Island, VA 23337 \\ willi.w.shur.1@gsfc.nasa.gov \\ Canan Bilen, $\mathrm{PhD}, \mathrm{CQE} \ddagger$ \\ Industrial \& Manufacturing Engineering Dept. \\ North Dakota State University \\ Fargo, ND 58105 \\ canan.bilen.green@ndsu.nodak.edu \\ Jerry Sterling, $\mathrm{PhD} \S$ \\ Balloon R\&D Laboratory \\ NASA - GSFC - Wallops Flight Facility \\ Wallops Island, VA 23337 \\ jerry.sterling@nasa.gov
}

\begin{abstract}
Design of safe and survivable structures requires the availability of guaranteed minimum strength thresholds for structural materials to enable a meaningful comparison of strength requirement and available strength. This paper develops a procedure for determining such a threshold with a desired degree of confidence, for structural materials with none or minimal industrial experience. The problem arose in attempting to use a new, highly weight-efficient structural load tendon material to achieve a lightweight super-pressure balloon. The developed procedure applies to lineal (one dimensional) structural elements. One important aspect of the formulation is that it extrapolates to expected probability distributions for long length specimen samples from some hypothesized probability distribution that has been obtained from a shorter length specimen sample. The use of the developed procedure is illustrated using both real and simulated data.
\end{abstract}

Key words: stratospheric balloons, minimum strength thresholds, extreme value distributions, factor of safety, ULDB, PBO cord.

\footnotetext{
* ALAA Member, Balloon Research Engineer

$\dagger$ Corresponding Author

$\ddagger$ Assistant Professor of Industrial and Mfg. Eng.

$\S$ AIAA Member, Materials Engineer
}

\section{INTRODUCTION}

The designer of a structure and the structural analyst aim to ensure that structures survive deployment and worst-case service life, and do not pose hazard during consequent decommissioning, disassembly, and final disposal. Additionally, in cases where extreme event failure must be anticipated, both the designer and the analyst must assure that the structure or system of interest fails in a safe fashion. These goals require the analyst to bound future events to perceived safe performance of the structure. Typically, these goals are achieved by assessing worst-case scenarios and by conservatively considering ageing of the structure. The qualification process typically entails the comparison of analytically obtained performance quantities to some thresholds. Uncertainty, on either of these requires the use of some form of factors of safety. The performance comparison is only as good as the analytic predictions of the performance quantities and the quality of the thresholds. The analytical predictions generally rely on the mechanical properties of the structural materials.

Reliance on strength thresholds for structural components or for structural materials is only warranted when such strength thresholds are guaranteed minima with some confidence level. Such thresholds exist for traditional structural materials with a long history of successful industrial applications. Quality controls, instituted by manufacturers and safeguarded by standards promulgated by industry wide umbrella organizations, provide assurance that these thresholds

1 
are indeed met and can be relied on. Nevertheless, the last three decades saw an ever more rapid introduction of new industrial materials with scant mechanical characterization and limited understanding of performance. Despite promising mechanical properties that suggest structural and economic efficiency many such materials usually experience a long route before being used in structural systems where failure has serious consequences. The early use of these new materials in not-so critical applications builds confidence. It allows industry and academia to collect information that eventually leads to an adequate understanding of the material, which is necessary for critical applications. In this paper we present a procedure for determining a minimum threshold with a desired degree of confidence for structural materials with none or minimal industrial experience.

\section{BACKGROUND}

The design, service-life qualification, and reliability predictions for current stratospheric balloons are based on merit-type assessments. Generally, these assessments do not compare realistic local stress projections to material performance parameters. Large safety factors are typically used to compare strength and service state indexes. This practice obscures the actual strength margin variation and is a hindrance to the design of weight-efficient structures. Analytical approaches that allow accurate predictions of stress resultants in the structural elements of the pneumatic envelope have been developed over the last fifteen years. 1, 2 However, until recently they had little influence on the design of balloons. With the development of the pumpkin shape super-pressure balloon and particularly with the use of a statically determinate design concept, accurate predictions for the stress resultants for the design defining states are available and safety factors more in line with the aerospace industry become applicable.

The lightweight superpressure balloon design for ultra long duration balloons (ULDB) uses load tendons made of a new, highly weight-efficient structural material, braided PBO cord. Mechanical strength characterization and industrial experience for this material has so far been inadequate for developing reliable strength thresholds for these load tendons. Extensive tensile strength testing of the cords and suitable evaluation of the test results is required. ${ }^{3}$

The present super-pressure ULDB design has about 300 load tendons with each load tendon about 500 feet long. The load tendons are statically determinate. There is no redundancy. Therefore, if any of the tendons fail, the balloon system fails. Allowing only one balloon in a fleet of 100 balloons to fail corresponds to determining a minimum strength threshold for $15,000,000$ feet long load tendon with a high degree of confidence. Typically, the specimen .length for initial strength characterization was 6 feet. That is, the length for which a minimum strength threshold is to be guaranteed with some acceptable confidence is $2,500,000$ times the length of the test specimen.

A guaranteed minimum strength threshold is vital to the successful deployment and survival of the superpressure balloon. We next look at the process of determining guaranteed minimum strength thresholds from test data.

\section{MINIMUM STRENGTH THRESHOLDS}

The determination of ultimate tensile strength characteristics of a material involves testing of specimens to failure. The entire population cannot be tested, as no material would be left for the technological purpose of interest. Typically, the high cost of the testing as well as of the material requires statistical strength characteristics to be estimated from a very small number of specimens. Statistical strength characteristics are determined by constructing from the sample an estimating function for the probability characteristics of the population.

It is often sufficient to use an "off-the-shelf" distribution to match the histogram of the available test data over the regime where data is available. Probability distributions typically used in characterization of strength in structural materials are the Gaussian distribution and the Weibull distribution. The Gaussian distribution is symmetrical, while the Weibull distribution is non-symmetrical. Tail ends of the Gaussian distribution extend to infinity, while the three-parameter Weibull has a finite left tail and infinite right tail. Generally, the probability density functions are chosen to match the data in the "cluster region" well, for which either the Gaussian or the Weibull distribution may serve adequately. But if the technology-query concerns the probability characteristics of the tails, such a characterization with either of these popular probability density functions may be misleading or strictly nonsense. Clearly, negative strength is not defined and there is no structural material with infinite strength. Alarmingly, the accurate characterization of the left tail is exactly what is required for the design of safe and survivable structures. 
What has been done too often in the past is to use of a $\mathrm{k \sigma}$ rule $^{4,5}$ to determine a minimum strength threshold (MST), namely

$$
\operatorname{MST}=\mu-k \sigma,
$$

where $\mu$ is the known mean and $\sigma$ is the known standard deviation of the material strength distribution, and $\mathrm{k}$ is usually taken to between 3.0 and 4.0. This threshold works well for established structural materials that follow a Gaussian distribution in the cluster region but for which experience indicates that the lower tail is void from these limits on. There are several criticisms to this practice. First, for strength failure data the Weibull distribution is more applicable than the Gaussian distribution, although there are other distributions that may empirically as well as physically be more appropriate. ${ }^{7,8}$ The MST ko rule has only a specific meaning for the contents of the left tail in the case of a Gaussian distribution. The $k$ values of 3.0 and 4.0 imply failure rates in the order of $10^{-3}$ and $10^{-5}$, respectively. Clearly, the same explanation cannot be given in the case of the Weibull or Gumbel distributions (Table 1).

\begin{tabular}{|c|c|c|c|}
\hline $\mathbf{k}$ & Gaussian & Weibull & Gumbel \\
\hline 3.0 & $1.35 \mathrm{E}-03$ & $6.40 \mathrm{E}-03$ & $0.46 \mathrm{E}-12$ \\
\hline 3.5 & $0.23 \mathrm{E}-03$ & $2.27 \mathrm{E}-03$ & 0 \\
\hline 4.0 & $0.03 \mathrm{E}-03$ & $0.70 \mathrm{E}-03$ & 0 \\
\hline 4.5 & 0 & $0.18 \mathrm{E}-03$ & 0 \\
\hline 5.0 & 0 & $0.01 \mathrm{E}-03$ & 0 \\
\hline 5.5 & 0 & 0 & 0 \\
\hline
\end{tabular}

Table 1. Enror in MST ko rule based on the Gaussian distribution.

When the Normal distribution is used incorrectly in place of the Weibul distribution, the MST for $\mathrm{k}=3.0$ would underestimate the failure rate as $1.35 \times 10^{-3}$, instead of the correct $6.4 \times 10^{-3}$. Similarly, if the Gumbel distribution applied but the Normal distribution was incorrectly substituted, then a significant overestimate of the failure rate for $\mathrm{k}=3.0$ would occur: $1.35 \times 10^{-3}$ instead of the much smaller $0.46 \times 10^{-12}$.

Second, typical test specimen are very small. Efficient design aims at nearly uniform exhaustion of available strength. This is seldom achieved even in aircraft structures. Still, in an efficiently designed aircraft structure there are significantly large regions that are projected to attain at least one time in their service-life stress levels near the available strength. So that may be even for a single aircraft a .1\% failure would make failure uncomfortably likely; in the case of a large fleet failure would be certain. The fact that aircraft designs are remarkably successful suggest that the probability distribution in use represents the left tail of the probability distribution of the actual population rather poorly and that there is some not too small strength level below which there is no content in the actual population. The somewhat careless use of the $k \sigma$ approach also renders any rational reliability assessment by statistical methods meaningless. Clearly, what is needed is a more careful characterization of the left tail of whatever probability distribution has been intuited from available sample data.

Thirdly, in a recent paper Annis ${ }^{6}$ has chastised the engineering community on their use of statistical methods. Failure probabilities computed for a normally distributed population by $\mu-k \sigma$ are dramatically underestimated. For instance, in the example of using a Normal distribution and expecting a failure rate in the order of $10^{-5}$, using $\mathrm{k}=4$ for this purpose, the failure rate is underestimated 6 fold if $99 \%$ confidence is required and the sample size is 31 when $\mu$ and $\sigma$ are unknown and must be estimated from the test data. This follows from applying the Student t-distribution, which provides corrections to the Normal distribution for sample size and confidence level when test data are also used to estimate the material strength mean and standard deviation. Even more severely, to achieve a failure rate in the order of $10^{-7}$ with about $99 \%$ confidence, $\mathrm{k}=6.7$ would be required when computing with the Normal distribution to obtain the corresponding correction provided by the Student $t-$ distribution. This fact has been generally ignored. Furthermore, it must be pointed out that the Student $t-$ is only applicable to test data that is distributed as normal. We next present a rational procedure for determining a guaranteed minimum strength threshold that correctly characterizes the lower tail behavior of material strength.

\section{A RATIONAL GUARANTEED MINIMUM STRENGTH THRESHOLD}

The GMST threshold procedure proposed here uses samples with different specimen lengths. It correctly identifies in some best sense an appropriate tentative strength distribution through tensile testing of a sufficiently large sample of the smaller-length specimens. The lower tail of the strength distribution may be truncated to ensure that the statistical strength distribution also makes physical sense. The identified tentative strength distribution is used to project a strength distribution for the longer-length tendons. The contradiction between these projections and the findings from the long-length specimens tests are used 
to deduce a correction to the hypothesized tentative strength distribution. Relatively small samples of various longer-length specimens are used to correct the projected strength distribution as necessary.

The universe of discourse is a uniform cable of infinite extent. Uniformity as meant here is nominal rather than strict. Usual variations due to fabrication processes that cause strength to vary along the length of the cable are accepted within that meaning. The goal is to determine a guaranteed minimum ultimate tensile strength for that cable. Typically a sample of constant length cable segments is tested. Let that length be $l$. The need of such practical aspects as loss of cable lengths to the gripping of the specimen is ignored in this discussion. Specimen length $l$ as discussed here.refers to the length of the test section alone. Assume that for a suitable sample size some probability density function $f_{\lambda}(\tau)$, where $\tau$ is the breaking or ultimate tensile strength of a particular specimen, has been determined to fit the data well. The probability that a specimen from the general population (each specimen of length $l$ ) will fail at or below the tensile load $\tau$ is

$$
F_{l}(\tau)=\int_{-\infty}^{\tau} f_{l}(\xi) d \xi
$$

Even if the hypothesized probability density function fits the data in the cluster region well, it is in general doubtful that its tails fit the statistical characteristics of the population. Clearly, few structural materials have regions in which the strength exceeds the average (mean) strength by say a factor of two. Yet for both the Gaussian distribution and the Weibull distribution the upper tail end is not void. Here we are concerned with the lower tail end.

Consider taking a second sample with specimens of length $\mathrm{N} l$, where $\mathrm{N}$ is a sufficiently large integer. We can easily assert that $\mu_{N} \leq \mu$ and $\sigma_{N} \leq \sigma$, because the probability of critical flaw inclusion increases with length. Here $\mu$ is the expected value or mean, and $\sigma$ is the standard deviation or the square root of the second moment about the mean.

Designating the probability of failure at or below a tensile load $\tau$ of a specimen of length $l$ by $F_{r}(\tau)$ one obtains the probability of failure at or below a tensile load $\tau$ of a specimen of length $\mathrm{N} l$ as

$$
\begin{aligned}
F_{N /}(\tau) & =\operatorname{Pr}(\text { each } l-\text { length strength } \leq \tau) \\
& =f_{i}^{\mathrm{N}}(\tau)+\sum_{j=1}^{\mathrm{N}-1}\left(\begin{array}{l}
\mathrm{N} \\
j
\end{array}\right) f_{i}^{j}(\tau)\left[1-f_{i}(\tau)\right]^{\mathrm{N}-j} \\
& =1-\operatorname{Pr}(\text { each } / \text { - length strength }>\tau) \\
& =1-\left[1-F_{l}(\tau)\right]^{\mathrm{N}}
\end{aligned}
$$

where the $\left(\begin{array}{l}N \\ j\end{array}\right)$ are the binomial coefficients. Subsequently, the probability density function for failure of the specimens of length $\mathrm{Nl}$ is given by

$$
\begin{aligned}
f_{N}(\tau) & =\frac{d}{d \tau} F_{N d}(\tau) \\
& =N f_{i}(\tau)\left[1-F_{i}(\tau)\right]^{N-1}
\end{aligned}
$$

The probability density function for failure of the specimens of length $\mathrm{N} l$ is the probability density function of the smallest order statistic ${ }^{9,10}$ (minimum value in a sample). In other words, the probability of failure at or below a tensile load $\tau$ of a specimen of length $\mathrm{N} l$ is equivalent to the probability of failure at or below a tensile load $\tau$ of the weakest specimen of length $l$ :

$$
\begin{aligned}
F_{N}(\tau) & =\operatorname{Pr}\left(\min \left\{X_{1}, X_{2}, \ldots, X_{N}\right\} \leq \tau\right) \\
& =\operatorname{Pr}\left(X_{(1)} \leq \tau\right),
\end{aligned}
$$

where $X_{1}, X_{2}, \ldots X_{N}$ are material strengths of each of the l-length segments that form the $\mathrm{N} l$-length tendon and $X_{(1)}$ is the strength of the weakest segment or the smallest order statistic. The limiting distribution function meaning as $\mathrm{N} \rightarrow \infty$, of the smallest order statistic, also known as extreme value distributions, falls into three families:

Type 1:

$$
\operatorname{Pr}[X \leq x]=\exp \left\{-e^{-(x-\xi) / \theta}\right\}
$$

Type 2:

$$
\operatorname{Pr}(X \leq x)= \begin{cases}0, & x<\xi \\ \exp \left\{-e^{-(x-\xi) / \theta}\right\}, & x \geq \xi\end{cases}
$$

Type 3:

$$
\operatorname{Pr}(X \leq x)= \begin{cases}\exp \left\{\frac{\xi-x}{\theta}\right\}^{k}, & x \leq \xi \\ 1, & x>\xi\end{cases}
$$

where $\xi, \theta(>0)$, and $k(>0)$ are parameters. The type 1 distribution is also referred to as the Frèchet-type distribution; the type 3 distribution is a Weibull-type

4 
distribution; and the type 1 distribution a Gumbel-type distribution. ${ }^{11,12}$ The Weibull and Gumbel distributions are the most commonly used extreme value distributions.

The tentative distribution allows the construction of a probability distribution for a population of larger test specimens, referred to as the projected distribution above. Typically, the larger-length specimen distribution has a lower mean and a smaller dispersion.

Development of tentative distributions for longer-length specimens from the distribution of smaller-length specimens is illustrated in Figure 2. The first plot assumes a tentative Gaussian distribution of $l=1$ ' length specimen strength. Strength distributions for $\mathrm{N} l$ $=10^{\circ}, 30^{\circ}$, and $40^{\circ}$ specimens are projected using (4) with $f_{l}(\tau)$ Gaussian. As the specimen-length gets larger, i.e. $\mathrm{N} \rightarrow \infty$, the projected distributions reach a limiting distribution. For $\mathrm{N}=30^{\circ}$ and $40^{\circ}$, projected strength distributions $f_{30}(\tau)$ and $f_{40}(\tau)$ practically overlap. Same observations hold when $f_{l}(\tau)$ is Weibull, Gumble, and others. Figure 1 also verifies that as the specimen length gets larger, its average strength as well as its standard deviation decreases.

In some cases, the tentative distribution fitted to the small-length test specimens may not make physical sense, such as an unbounded right tail indicating an infinite material strength. Although, statisticians would argue that an infinite right and/or left tail, as in the Gaussian distribution, is of no concern since even $\pm 3 \sigma$ would cover $99.72 \%$ of the population. However, as discussed by Annis $^{6}$ in probabilistic failure rate calculations of $10^{-7}$ or smaller, accuracy can be highly in error.

Additionally, an off-the-shelf probability distribution that might have been chosen and suitably matched to the data in the cluster region will not be able to characterize the statistical content of the outlying extremes in the population of strength test specimen of a real material. Given a hypothesized probability distribution that fits the data in the cluster region well one would expect that $f_{\mathrm{N} l}(\mu)$ and $F_{\mathrm{N}}(\mu)$, where $\mu$ is the mean tensile strength of the $l$ - length specimen sample, will be confirmed approximately by a sufficiently large $\mathrm{N} l$ sample. However, at the lower tail end where probability of failure $p$ is small, say $f_{l}\left(\tau^{\prime}\right)=.01$, we expect contradictions. For this discussion let $\mathrm{N}=10$. Then for the strength value $\tau$ ' the probability of failure of an $\mathrm{N} l$ specimen is dominated by the first term of the sum and estimated to be slightly larger than $(.904)(10)(0.01)=.094$, i.e. one would expect from the formula that about every $11^{\text {th }}$ specimen to fail at or below $\tau^{\prime}=$ inverse $\left\{f_{l}(\tau) \mid p=.01\right\}$. If our contention is correct, however, then failure at or below $\tau$ ' would be considerably less frequent. Furthermore, one might suspect that there is some useful information in the discrepancy between the projected distribution for the $\mathrm{N} l$ sample $\left(f_{\mathrm{N} l}\right.$ and $\left.F_{\mathrm{N} l}\right)$ and a tentative probability distribution that could be constructed from the $\mathrm{N} l$ sample data.
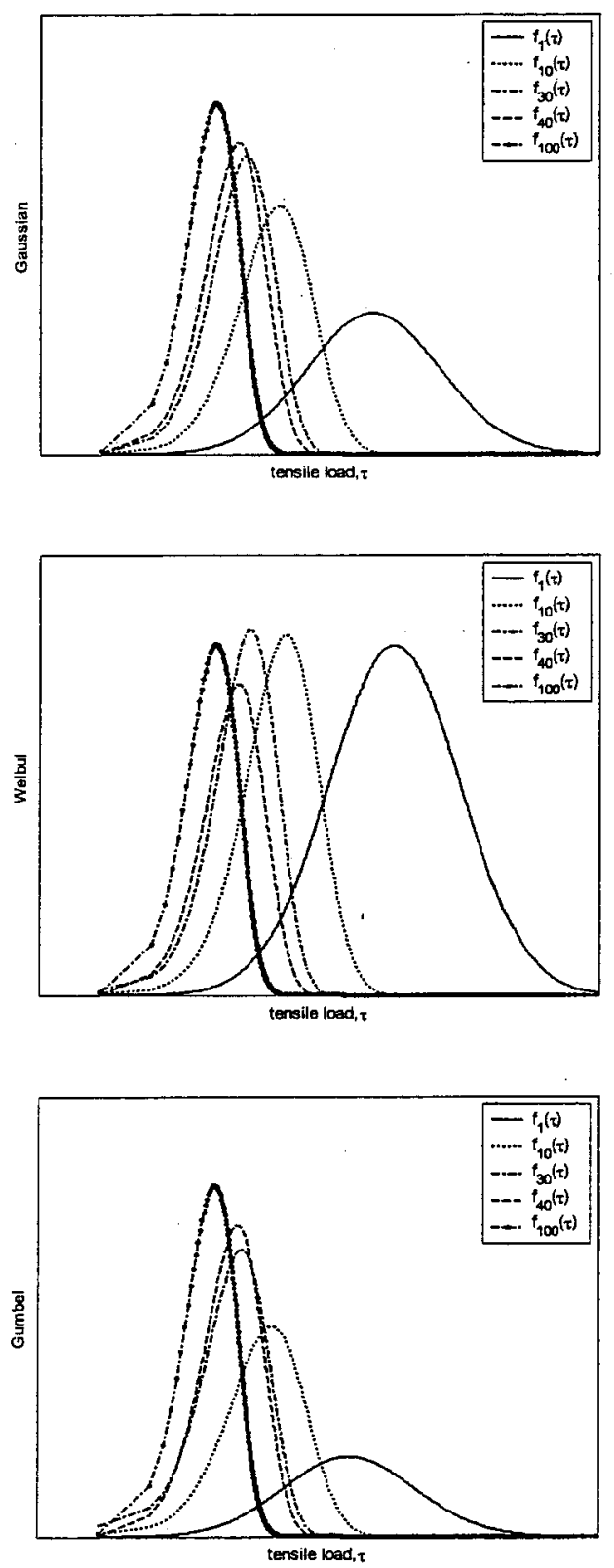

Figure 1. Projected breaking strength distributions for various lengths and statistical distributions. 
Tentatively Postulated Probability Distribution Modification

When modifying some assumed probability distribution to better represent certain probabilistic characteristics of a population, it is still necessary to make sure that the modified representation is a probability distribution. For the probability density function $f(x)$ it means that $f$ is strictly non-negative and that

$$
\int_{-\infty}^{+\infty} f(\xi) d \xi=1.0
$$

In the case that a chosen probability density function fits the data in the cluster region very well but outside some regime it is known that the actual distribution is void while the chosen function is non-void one could use simple scaling to affect a suitable correction to the originally chosen probability density function. Say reality dictates $f(\mathrm{x}<\mathrm{a})=0$ and $f(\mathrm{x}>\mathrm{b})=0$, then one can construct

$$
\begin{gathered}
f(x)= \begin{cases}f(x) / \alpha, & a \leq x \leq b \\
0, & \text { otherwise }\end{cases} \\
\text { where } \alpha=\int_{a}^{b} f(\xi) d \xi .
\end{gathered}
$$

density function $f(x)$ fits the population characteristics within the cluster region well, and matches the requirement that outside the range $[\mathrm{a}, \mathrm{b}]$ the probability density is void.

\section{Projected Distribution Corrected Through Test Data}

In order to obtain an estimate for a guaranteed minimum strength threshold, it is necessary to probe the lower tail of the probability distribution. The premise is that the projected distribution will deviate significantly from a distribution that would be constructed for the sample test data of the large-scale specimen. Accepting then the shape of the tentative distribution in both the cluster region and the right tail as adequate for the small specimen population one can rapidly produce modifications to the projected distribution for the large specimens population given today's computer technology. This can be done by recalculating the projected distribution from modifications to the tentative distribution for the small-scale specimen population. The modifications would be made to the lower tail end of the tentative distribution by assuming some portion of its lower section void, and in addition, by corresponding re-scaling in the non-void range of the probability density function. The modification that produces the best data-fit between the projected distribution and the data of the large-scale specimen population is then accepted as an improvement to the probability distribution for the small-scale specimen population.

\section{Illustrative Example}

A vital piece in the design of the lightweight superpressure ULDB is accurate characterization of the strength of the tendon material. The tendons are made of a new polymeric engineering material for which little industrial experience exists, requiring extensive tensile strength testing of the cords. Specimen length for initial strength characterization has been around 6 feet.

In Figure 2 Weibull distributions provided the best fit to data from in-production testing of the tendon material (6.2' specimens) by the manufacturer in early 2003 . The Sample 1 specimens are from the first quality control test of spools 1-75 during the fabrication of the balloon. Similarly, Sample 2 specimens are from the first quality control test of spools 7.6-143. Sample 3 and 4 are from the second quality control tests for spools 1-75 and 76-143 respectively. The Weibull parameters are

$$
\begin{aligned}
& \text { Sample 1: } \delta=1590, \alpha=11.5, \beta=1460, \\
& \text { Sample 2: } \delta=2060, \alpha=7.34, \beta=1110, \\
& \text { Sample 3: } \delta=53, \alpha=21.1, \beta=2780 \text {, and } \\
& \text { Sample 4: } \delta=1550, \alpha=10.2, \beta=1570 .
\end{aligned}
$$

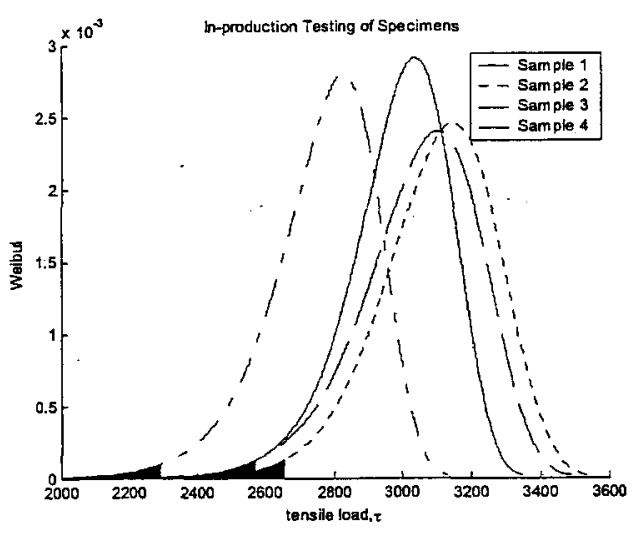

gure 2. In-production strength testing of specimens of about $6^{\prime}$ long. The shaded area for each sample depicts minimum threshold value for approximate 0.01 failure probability. 
Figure 3 shows similar fitted distributions to Sample A $\left(6.2^{\prime}\right)$ and Sample B (26.2') for testing data obtained in August 2003. Both follow a three-parameter Weibull distribution with parameters

$$
\begin{aligned}
& \text { Sample } A: \delta=2680, \alpha=3.5, \beta=293 \text { and } \\
& \text { Sample B: } \delta=1710, \alpha=12.6, \beta=1280 .
\end{aligned}
$$

Therefore, parent distribution of the material strength, regardless of tendon length, can be assumed to be distributed as a three-parameter Weibull with probability distribution function

$$
f(x)=\frac{\alpha}{\beta}\left(\frac{x-\delta}{\beta}\right)^{\alpha-1} \exp \left[-\left(\frac{x-\delta}{\beta}\right)^{\alpha}\right] \text { for } x>\delta,
$$

and with probability distribution function

$$
F(x)=1-\exp \left[-\left(\frac{x-\delta}{\beta}\right)^{\alpha}\right] \quad \text { for } x>\delta,
$$

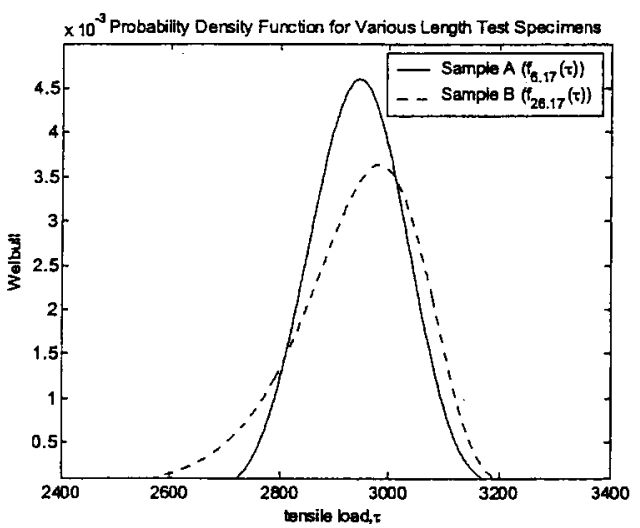

Figure 3. Weibull fitted probability density functions to the various length test specimens.

where $\delta$ is the location parameter, $\alpha$ and $\beta$ are shape and scale parameters, respectively.

We observe (Figure 3) that the outcome of the experiments contradicts what would rationally be expected. We expect the mean for Sample B to be less than the mean for Sample $A$, and we expect the variance (dispersion) of Sample $B$ to be significantly less than for Sample A, as longer specimens will have more critical flaws (see Figure 1). This leads to the conclusion that either the sample sizes are too small or that the samples have come from two different populations. In our case, due to diligent experimental controls, we are certain that the samples came from the same population. Therefore we conclude that larger sample sizes are needed to properly carry forth the quantitative assessment of minimum strength guarantee. However, once further experiment results are in hand, the procedure will be as follows below.

Let $X_{1}, X_{2}, \ldots, X_{N}$ denote the material strengths of each of the $l$-length segments that form the $\mathrm{N} l$-length tendon. From (5),

$$
\begin{aligned}
F_{N} & =1-\operatorname{Pr}\left(X_{(1)}>\tau\right) \\
& =1-[\operatorname{Pr}(X>x)]^{N} \\
& =1-\exp \left[-\left(\frac{x-\delta}{\beta}\right)^{\alpha}\right]^{N} \\
& =1-\exp \left[-\left(\frac{x-\delta}{\beta}\right)^{\alpha N}\right]
\end{aligned}
$$

Therefore, given that the strength distribution of the $l$ length specimen is a Weibull distribution with parameters $(\delta, \alpha, \beta)$, the parameters of the $\mathrm{N}$-length specimen are given by $(\delta, N \alpha, \beta)$. But as we expected the data will not cooperate easily as we do not see such a relation between the parameters of Sample $A$ and Sample B. (Sample A: Weibull $(\delta=2680, \alpha=3.5, \beta=$ 293), Sample B: Weibull $(\delta=1710, \alpha=12.6, \beta=$ 1280)).

Which means that the distribution of the $\mathrm{N} l$-length specimens for this particular material is not simply in the form of $\min \left\{\mathrm{X}_{1}, \mathrm{X}_{2}, \ldots, \mathrm{X}_{\mathrm{N}}\right\}$ as derived in (5). Hence, the projected distribution of $\mathrm{N} l$-length specimens is corrected using the available test data. The $\mathrm{N} l$-length strength is distributed as

$$
\mathrm{Y}_{\mathrm{N} l}=a^{\mathrm{N}} \cdot \min \left\{\mathrm{X}_{1}, \mathrm{X}_{2}, \ldots, \mathrm{X}_{\mathrm{N}}\right\}-b^{\mathrm{N}}
$$

with probability distribution function

$$
\begin{aligned}
F_{N}(\tau) & \doteq F_{x}\left(\frac{y+b^{N}}{a^{N}}\right) \\
& =1-\exp \left[-\left(\frac{y+b^{N}-a^{N} \delta}{a^{N} \beta}\right)^{\alpha}\right],
\end{aligned}
$$

where $a, b$, and $k$ are positive. Therefore, given that the strength distribution of the $l$-length specimen is Weibull with parameters $(\delta, \alpha, \beta), \mathrm{N} l$-length specimen are also distributed as Weibull with parameters $\left(b^{\mathrm{Nk}}-\mathrm{a}^{\mathrm{N}} \delta, N \alpha\right.$, $\mathrm{a}^{\mathrm{N}} \beta$ ). Such $a$ and $b$ exists for the available data. 
Therefore, we can predict the distribution of the $26^{\prime}$ specimens from the distribution of the $6^{\prime}$ specimens. Hence we can also predict the distribution of the $500^{\circ}$ specimens.

Once, the correct" distribution of the Nl-length specimen, $Y_{\mathrm{N} l}$, is identified, the guaranteed minimum strength threshold $T_{p}$ is the threshold value that contains at most proportion $\mathrm{p}$ of the $\mathrm{N} l$-length strength population with $100(1-\alpha) \%$ confidence. Then,

$$
\operatorname{Pr}\left(Y_{N}<T_{p}\right)<p
$$

with $100(1-\alpha) \%$ confidence, where $p$ is the desired maximum proportion in the lower tail of the strength distribution (Figure 4). Exact and closed form solutions exist for various extreme value distributions ${ }^{13}, 14,15$ including the three-parameter Weibull distribution. ${ }^{16,17}$

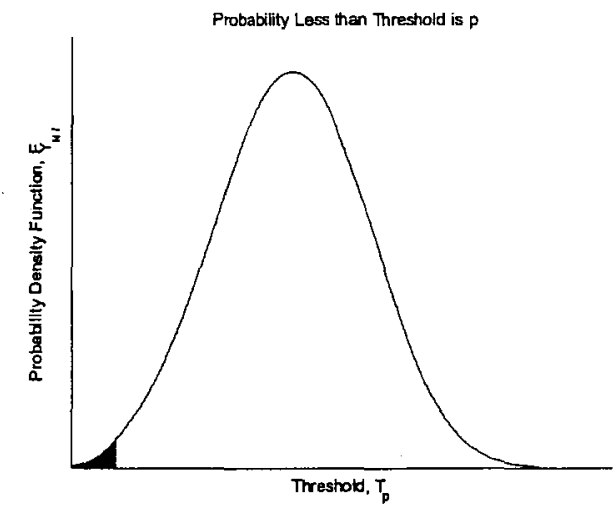

Figure, 4. With $100(1-\alpha) \%$, at most $100 \mathrm{p} \%$ of the tendons have strength greater than $T_{p}$.

Our test data have been limited to only two different lengths. Additional testing at various other lengths is necessary for accurate projection of the load tendon strength distribution. It is also important to address the variability that has been observed in different sample tests (Figures 2 and 3 ).

The procedure developed here assumes that the segments forming the load tendon have identical strength distributions as they are of the same length. For our particular material, preliminary tests indicate variability along the length of the cable ${ }^{3}$. Again, this variability needs to be taken into account for correct identification of a guaranteed minimum strength threshold for the load tendon.

\section{CONCLUSION}

This paper presented a rational approach for determining a guaranteed minimum threshold with a desired degree of confidence, for structural materials with none or minimal industrial experience. The procedure identifies an appropriate tentative strength distribution through tensile testing of sufficiently large sample of smaller-length specimens. The lower-tail of the strength distribution is truncated to ensure that the statistical strength distribution also makes physical sense. The tentative strength distribution is used to project a strength distribution for the longer-length tendons. Relatively, small samples of various longerlength specimens are used to correct the projected strength distribution as necessary. High-quality data is a crucial component in determining a guaranteed minimum strength threshold.

\section{REFERENCES}

[1] W.W. Schur, and J.M. Simpson, "Finite Element Solution for the Structural Behavior of a Scientific Balloon," COSPAR 90.

[2] F. Baginski and W. Schur, "Structural Analysis of Pneumatic Envelopes: A Vaiational Formulation and Optimization Based Solution Process," AIAA J., Vol. 41, No. 2, February 2003, pp. 304-311.

[3] W.J. Sterling, "Material Properties Analysis of Structural Members in Pumpkin Balloons," Paper No. 2003-6777, AIAA ATIO conference, Denver, November 2003.

[4] MIL-HDBK-5E, 1 June 1987

[5] MIL-HDBK-17 Composite Materials Handbook.

[6] C.E. Annis, "A Strategy for Improving 'Confindence' in a Failure Probability," Paper No. AIAA-2003-1572, $5^{\text {th }}$ AIAA NonDeterministic Approaches Forum, Norfolk, April 2003.

[7] W.Q. Meeker and L.A. Escobar, Statistical Methods for Reliability Data, WileyInterscience, New York, 1998.

[8] G.E.P. Box, W.G. Hunter, and J.S. Hunter, Statistics for Experimenters, John Wiley \& Sons, New York, 1978. 
[9] David, H. A. Order Statistics, 2nd ed. New York: Wiley, 1981.

[10]N. Balakrishnan and A.C. Cohen, Order Statistics and Inference. New York: Academic Press, 1991.

[11] N.L. Johnson, S. Kotz, and N. Balakrishnan, Continuous Univariate Distribuions, Volume 2, John Wiley \& Sons, New York, 1995.

[12] J. Galambos, The Asymptotic Theory of Extreme Order Statistics, John Wiley \& Sons, New York, 1978.

[13] J.F Lawless, "Construction of Tolerance Bounds for the Extreme-value and Weibull Distributions," Technometrics, Vol. 17, pp. 255-261; 1975.

[14] M. Engelhardt and L.L. Bain, "Simplified Statistical Procedures for the Weibull or Extreme Value Distribution," Technometrics Vol. 19, pp. 323-331, 1977.

[15] R. Dasgupta and D.K. Bhaumik, "Upper and Lower Tolerance Limits of Atmospheric Ozone Level and Extreme Value Distribution," Series B, Indian Journal of Statistics, Vol. 57, pp. 182-199, 1995.

[16] R.A. Jones and F.W. Scholtz, "Tolerance Limits for the Three Parameter Weibull Distribution, Mathematics and Modeling," Technical Report MM-TR-11, Boeing Computer Services Company, Seattle, WA, 1983.

[17] R. A. Johnson and J. H. Haskell, " $A n$ Approximate Lower Tolerance Bound for The Three-Parameter Weibull Applied to Lumber Property Characterization," Statistics \& Probability, Letters Vol. 2, pp. 67-76, 1984. 